\title{
The impact of differing door widths on passenger movement rates
}

\author{
N. G. Harris ${ }^{1}, \varnothing$. Risan $^{2} \&$ S.-J. Schrader ${ }^{2}$ \\ ${ }^{1}$ The Railway Consultancy Ltd., UK \\ ${ }^{2}$ Norwegian State Railways, Norway
}

\begin{abstract}
Increases in rail traffic are putting pressure on capacity, especially in older systems. In urban railways, capacity is determined by station stops, including the physical dimensions of trains and platforms, and the behaviour of passengers. Previous research has identified a range of factors which affect the rates at which passengers alight from, and board, trains, but train door width may not be as significant a factor as once thought. Using data from a worldwide set of operational surveys, this paper sets out a number of hypotheses to explain this, noting that other constraints (including difficulties in measuring passenger movement rates, the interior design of trains and (crucially) the positioning of boarding passengers on platforms) may be more important.
\end{abstract}

Keywords: rail transport, stations, planning, passenger behaviour.

\section{Introduction}

A resurgence in rail travel has led many urban railways to near their system capacity. This has particularly affected older systems, built 100 years ago or more, and to lower or smaller standards, examples including the Paris metro and London Underground; demand on the latter has increased $\approx 60 \%$ over the last 20 years, with only half of one line added to the 10-line network. As major investment is extremely expensive (schemes at Victoria and Bank stations in London are both understood to cost almost $\$ 1$ bn each), and funds limited, many operators have needed to pay much more careful management attention to passenger flows within stations and to/from trains. A "systems engineering" approach has been necessary, in order to avoid spending money on one bottleneck, only to see the problem transferred to an adjacent station or route. 
Part of the attention to this problem has been addressed at train design. Through gangways and fewer seats have become normal responses to maximising capacity within the train. However, line capacity in urban areas is significantly determined by station stops, at which the passenger contents of that capacity need to be exchanged. Here, there are physical limitations associated with passenger, vehicle and platform characteristics.

This paper concentrates on vehicle characteristics and, in particular, doors. These are often the bottleneck of station stops, since through these all boarders and alighters must pass, if a railway is actually to transport passengers from a to b. In fact, all that really matters is the critical or busiest door on a train, since movements at every other door take place simultaneously, and will (by definition) have completed before those at this critical door.

The paper is set out as follows. Section 2 contains a literature review of major contributions in this field. Section 3 describes the dataset available for this analysis, with section 4 setting out a summary of the modelling work undertaken on this dataset. Section 5 provides a discussion which helps to explain why our original hypotheses do not hold good, with section 6 providing our conclusions.

\section{Previous research}

A number of researchers have investigated the reasons which determine the rates at which passengers board and alight from trains. The three key types of factors which are relevant are:

(i) passenger characteristics (e.g. quantities, types of people, cross-flows);

(ii) station characteristics (e.g. platform widths, egress facilities); and

(iii) rolling stock characteristics.

That the detailed design of trains affects passenger movement rates is not in doubt: Figure 1 shows clearly the alighting rates from different types of rolling stock at Oslo's Nationaltheatret station, which is a busy central-area underground station with a single island platform in each direction. Types 69 and 72 are designed for inner-suburban traffic and the other train types for outer-suburban and long-distance traffic.

Although London Underground had identified door width to be a factor affecting their passenger movement rates, in operational research in the late 1980s [1], perhaps the foremost researcher in this field over the years has been Prof. Ullrich Weidmann at Zurich [2-4]. His 1994 paper identifies relationships with door width to be of the form:

$$
\text { Flow }=\mathrm{f}\left(\mathrm{d}^{-0.1}\right)
$$

where the exact value of the function depends upon door spacing, being larger for wider door spacing (as might be appropriate for a suburban railway with 2 doors per car, as opposed to a metro).

However, Heinz [5], working in Sweden, identified different types of passenger flow through train doors, including not only fully-simultaneous movement (where door widths permit it) but also a shoulder-to-shoulder variant (see Figure 2) which nevertheless provides a greater flow than a single column of passengers. Both 
types are also subject to the sorts of edge effects found in pedestrian planning of buildings such as stations, and suggest that an improvement may be needed to Weidmann's formula.

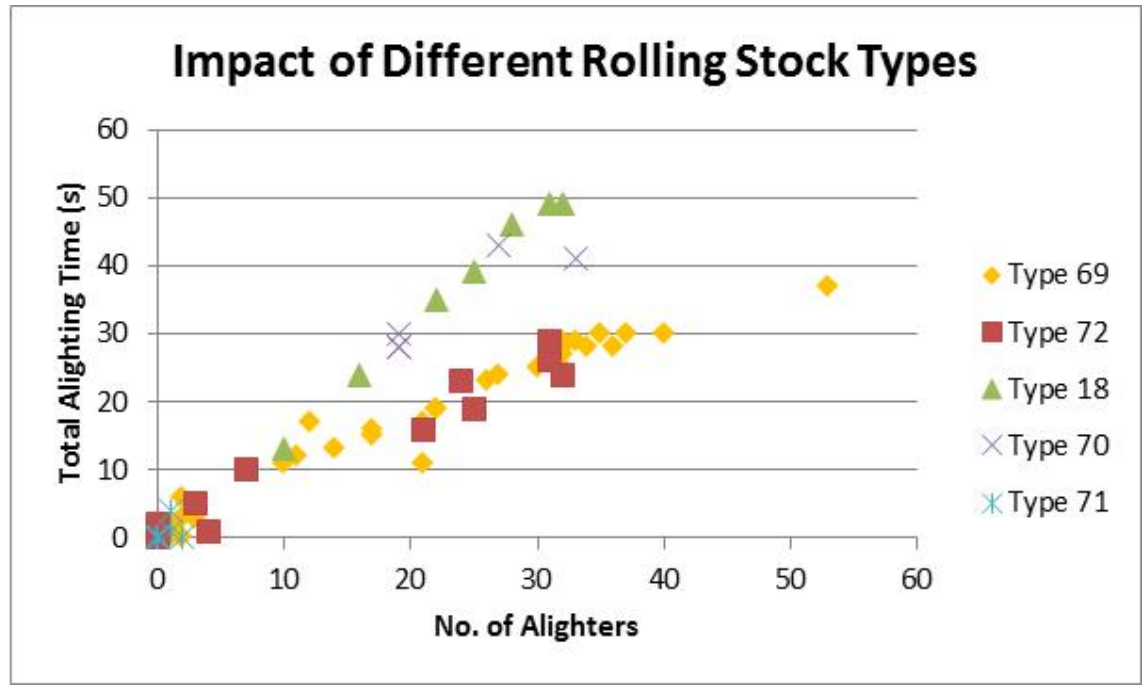

Figure 1: Impact of different rolling stock types on passenger alighting rates, Nationaltheatret station, Oslo. Source: own data.
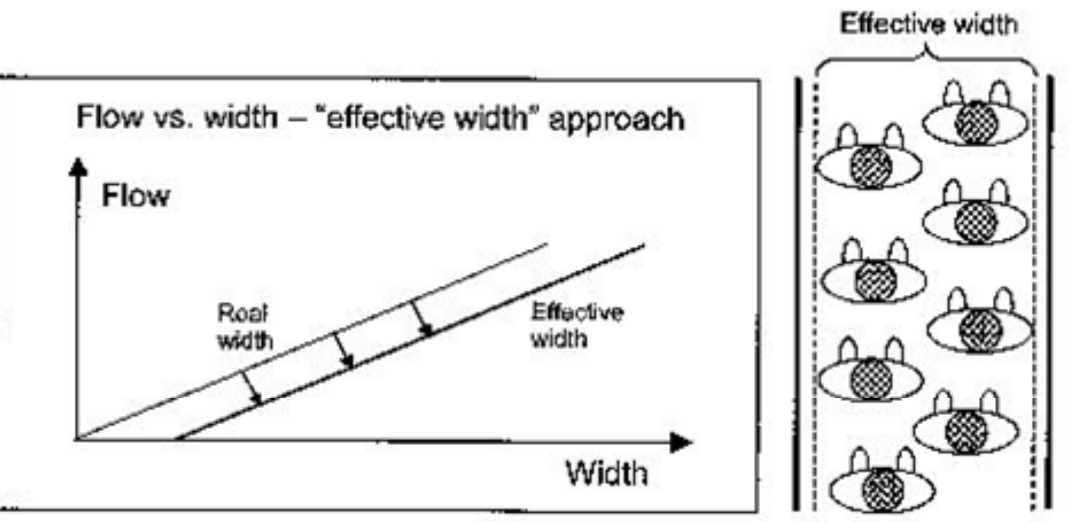

Figure 2: Shoulder-to-shoulder flow. Source: [5, Figure 30].

Both the existence of different types of flow, and of edge effects, strongly imply that the impact of door width on passenger movement through train doors is not linear. Nevertheless, it is still of keen interest to train operators to understand what this function is. 


\section{Data available}

The dataset used for this analysis is a database held jointly by the Railway and Transport Strategy Centre (RTSC) at Imperial College London, and the Railway Consultancy Ltd (RCL). The RTSC organises research programmes for the CoMET, Nova and ISBeRG metro and suburban railway benchmarking groups, and meetings to discuss key performance indicators and case studies of subjects of interest are usually accompanied by surveys. RCL undertakes a range of operational performance analysis for different railways around the world, and specific operational research studies have also been undertaken by RCL for the Tyne and Wear metro and Norwegian State Railways (NSB). The joint RTSC/RCL dataset therefore now includes over 125 surveys undertaken worldwide to a common methodology, with each one typically of 30 observations of trains calling at busy stations. Observations include detailed timings of processes, as well as the numbers of passengers (boarders, alighters, and those remaining in the vestibule without alighting); both timings and counts are limited to the busiest, or critical, door. Other measurements (e.g. door dimensions) are routinely taken at the time, or taken from scale drawings afterwards.

This dataset has been under development for 15 years, and a number of papers have resulted from it. These have included work on the stepping distance between the train and the platform, on vestibule design, boarding and alighting rates at high passenger volumes [6], comparative passenger movement rates [7], and impacts of particular elements of train and station design [8]. A comprehensive statistical analysis of the factors underlying passenger movement rates (in passengers/second) is ongoing.

However, one area of contention has been the impact of door width, as multiple linear regression has identified the impact to both statistically-insignificant and negative, whereas one would expect it to be strongly positive. This leads to two questions: (i) is it positive? and (ii) is it linear or not? Earlier work quoting passenger movement rates in pass $/ \mathrm{sec} / \mathrm{m}$ of door width implicitly implies that the impact of door width is linearly-positive, but perhaps that is not the case.

\section{Modelling}

At first sight, the ability to include 125 surveys, each representing 30 consecutive observations of peak trains, might make it appear straightforward to obtain definitive results. However, there are known to be many factors affecting passenger boarding and alighting rates, and sufficient data was collected on no less than 17 variables, which impacts on the number of degrees of freedom available within statistical modelling. Moreover, many of the relationships have been hypothesised (e.g. by [3] and [5]) not to be linear.

In order to avoid statistical problems associated with correlation and unknown functional forms, regression was therefore undertaken using a multivariate fractional approach applied to the 17 train, platform and passenger flow variables, and that work has been reported elsewhere [9]. This paper, however, concentrates 
on the explanation for one key result: that statistically-significant results could not be obtained for a variable which would intuitively be obvious as an explanator of passenger movement rates i.e. door width.

\section{Discussion}

One might expect reasonably passengers to board, and alight from, trains more easily if trains have bigger doors. However, it is not obvious as to the nature of this relationship: for instance, is it linear or not? This analysis therefore examines the dataset for the impact of door width upon these movement rates. Clearly, as expected, there is substantial variation due to a range of other factors, plus random noise from measurement error.

Heinz [5] postulated that, with narrow doors, passengers board and alight in "lanes", whilst when doors are wider, a more continuous flow is possible within an effective width which takes account of edge effects. The change-over from lane-based to continuous flow would be expected to be marked by an increase in the rate of flow per width (see Figure 3). Contrary to Heinz's view, however, our previous work at Eastleigh [6] showed that flow varied in three stages through a boarding or alighting episode, equivalent to lane-based, then continuous, then lane-based flow.

Figure 4 shows our results from the international dataset of the impacts of door width against the rates of passengers boarding and alighting. With rates shown in units of passengers/second, there are clear steps corresponding to the ability of passengers to undertake simultaneous movements.

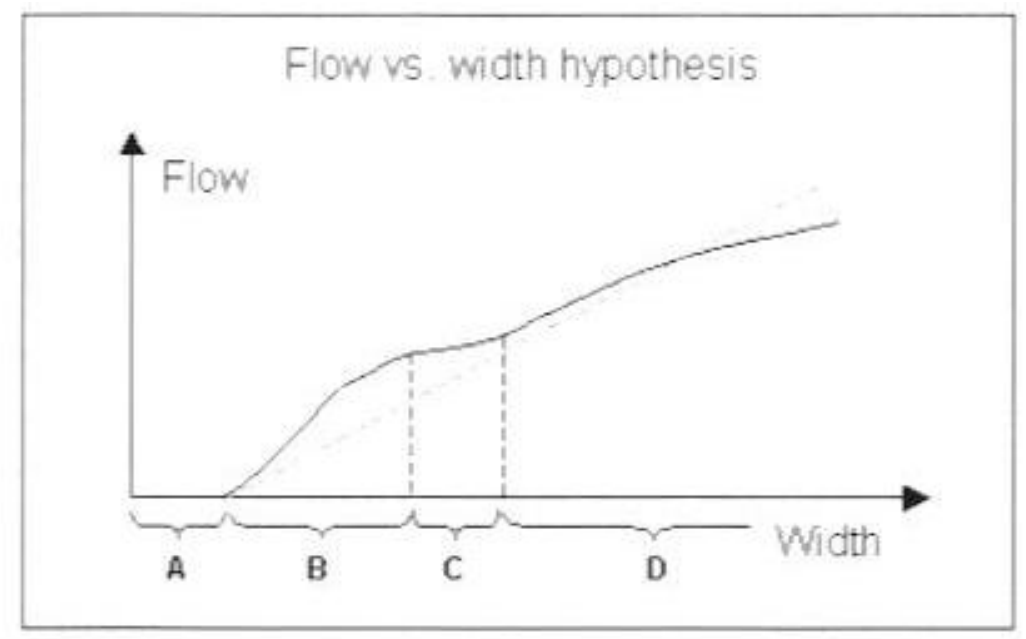

Figure 3: Expected relationship between passenger flow and door width. Source: [5, Figure 31]. 
The first conclusion from this analysis is therefore that the ability of passengers to undertake simultaneous movements is critical in enabling doors to function well. At a door width of around $1.25 \mathrm{~m}$ (a common design), flow is maximised, as it enables two passengers to alight side by side. Boarders, on the other hand, seem more able to board where one person follows the shoulder of another, so that continuing increases in door width remain helpful, if only to a minor degree.

Increasing door width therefore clearly does not lead to a proportionate increase in passenger alighting rates (see Figure 4). But it is also helpful to understand the behavioural and cultural reasons why this might be. Boarding is often not a wellregulated affair (see Figure 5).

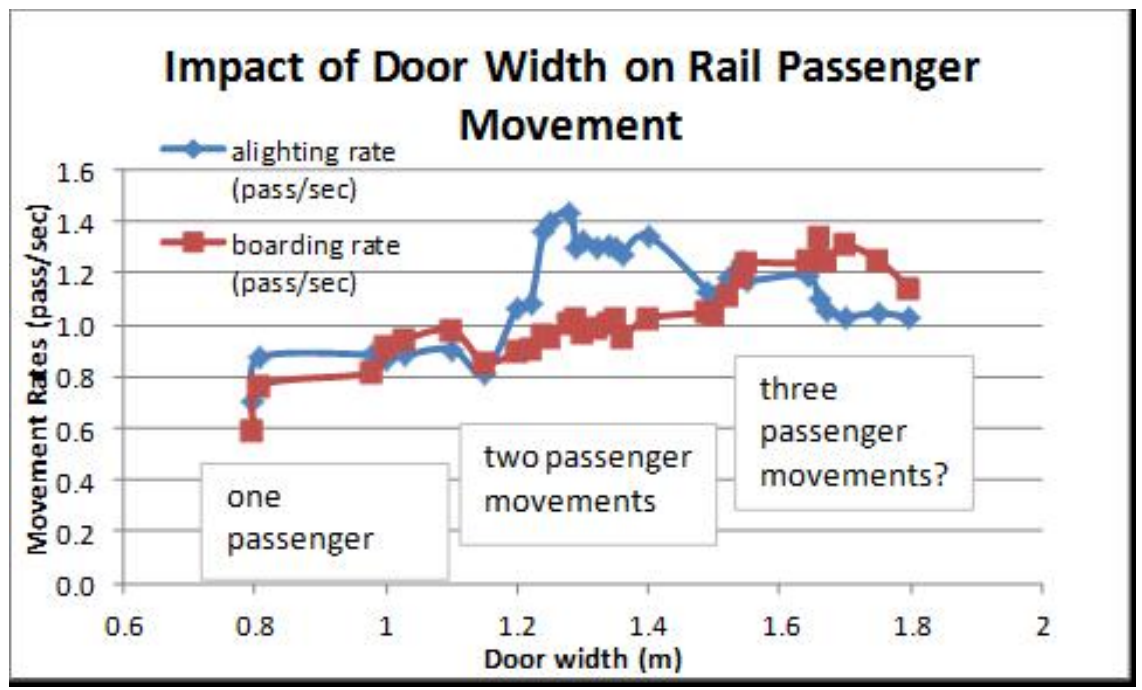

Figure 4: Impact of door width on rail passenger movement. Source: own analysis of joint RTSC/RCL database.

However, some boarding passengers, supported by pro-active platform management, can be encouraged to stand aside (especially in more organised cultures such as Singapore, Taiwan and Japan), with the aim of speeding up the alighting process. Nevertheless, detailed observation of this process shows that it is not entirely successful. The key problem is that the gap left by passengers attempting to board is often narrower than the width of the door, so the design flow rate of the door is not achieved. When considered as an overall process, the bottleneck is no longer the door-frame, but the space on the platform. Earlier researchers (e.g. [5]) noted that the width of the aisle left by boarding passengers was narrower than the door width, but we believe the position to be more serious than this. Our behaviourally-based hypothesis is that the first potential boarders stand aside, subject to leaning in a few centimetres so that they can see when the flow of alighters is likely to end. Subsequent boarding passengers, if they want to see, then have to stand a few centimetres further forward. This continues until the 
boarders at both sides of the door more or less meet, almost preventing anyone alighting at all (see the observed behaviour in Figure 5 and the hypothesised behaviour in Figure 6). This suggests that an easier ability to see into trains might reduce the propensity to stand in the way. In the meanwhile, urban rail operators continue to try a range of measures such as announcements, platform markings

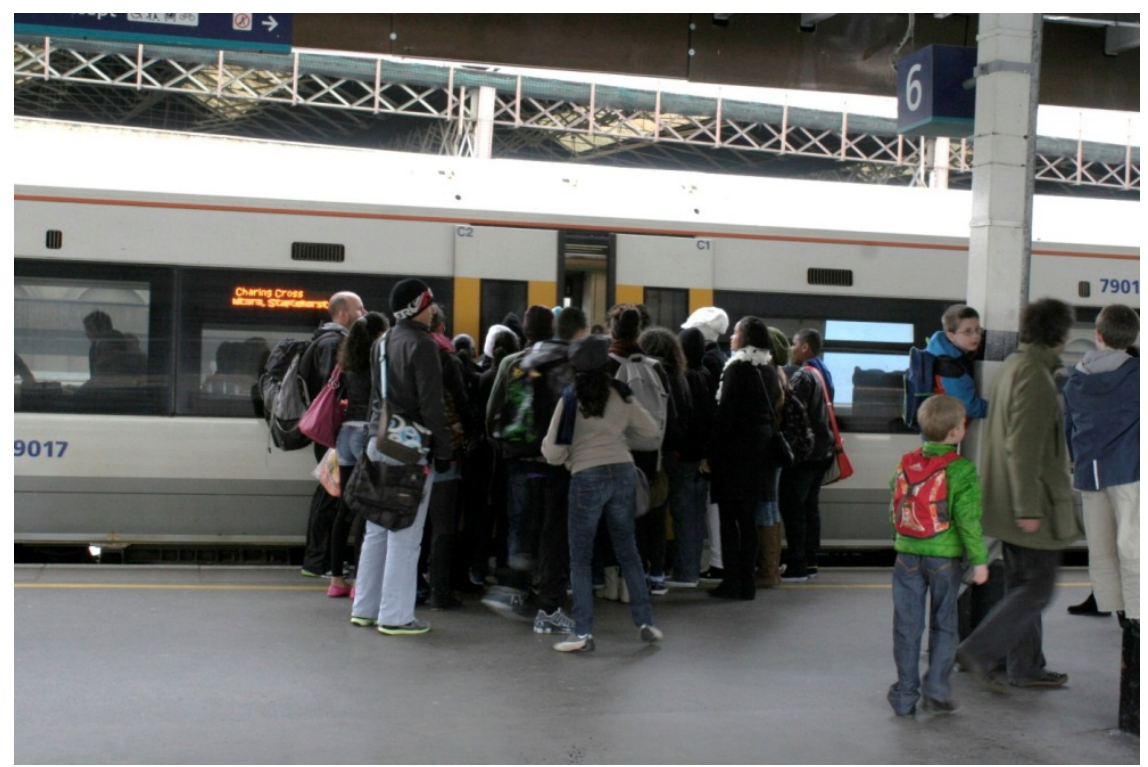

Figure 5: Unmanaged Passenger Boarding Behaviour, London Bridge, UK.

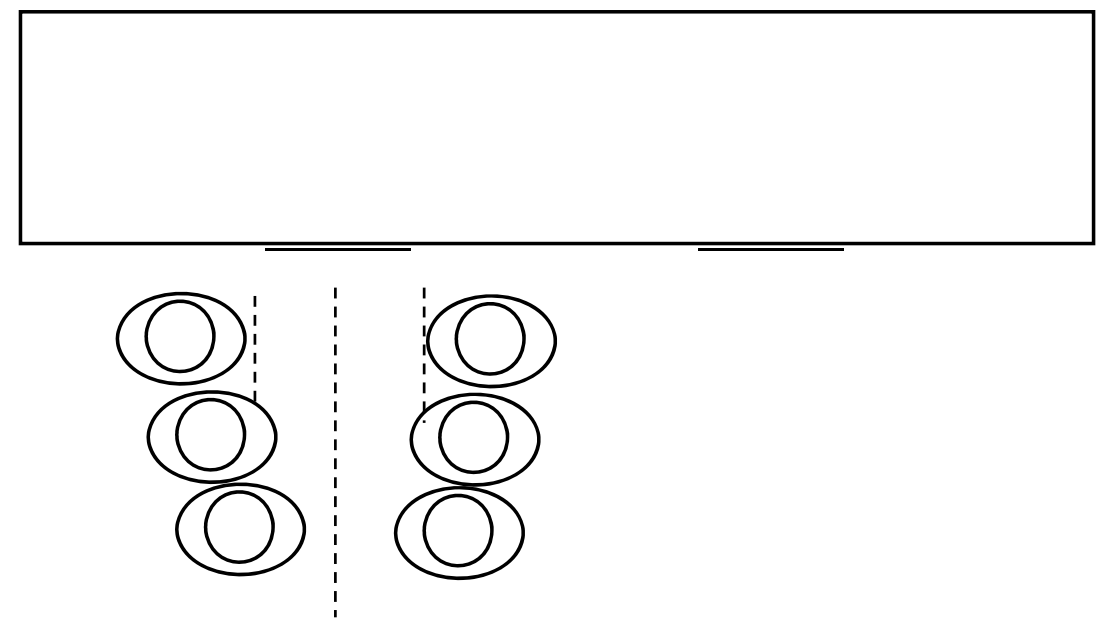

Figure 6: Hypothesised behaviour of boarding passengers. 
and posters (see Figure 7) to achieve behavioural change. An analysis of residuals from other modelling [9] is inconclusive in assessing evidence that railways in more organised cultures are achieving higher passenger flow rates.

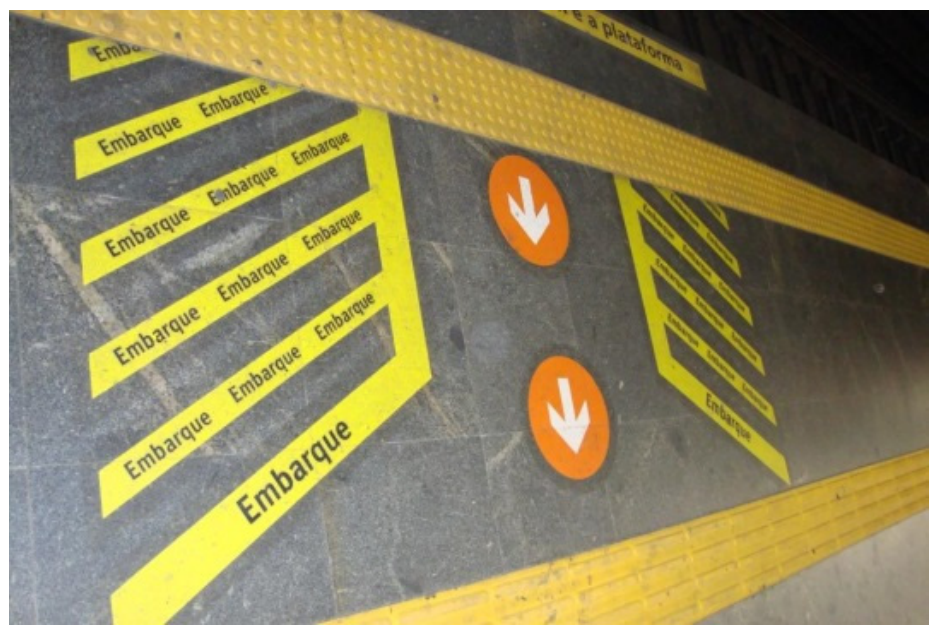

(a)

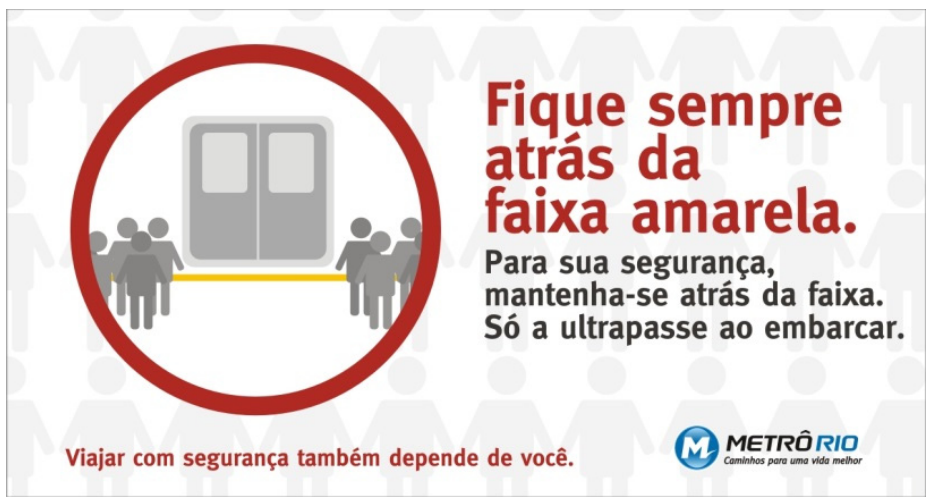

(b)

Figure 7: Examples of measures to encourage boarders to stand back. (a) Platform markings; (b) Posters.

The above analysis suggests that use of threshold values might be helpful in determining passenger movement rates through train doors, with a key threshold being a width of about $1.25 \mathrm{~m}$ range. However, the achieved performance is not necessarily a function of actual door width, but of the width of the corridor left on the platform, which is rather more difficult to manage (or even measure). Observations show that the shape of the "funnel" of passengers varies, but that the corridor left free remains similar at its narrowest point, permitting little more than one passenger wide. Figure 8 shows this hypothesis. 

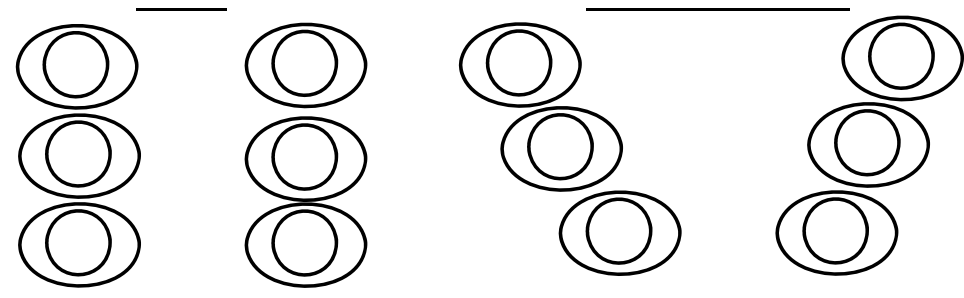

Figure 8: Possible "funnel" shapes for (left) narrow and (right) wide doors.

With even wider doors, the evidence apparently shows a reduction in passenger movement rate, but this may have other explanations. For instance, the real bottleneck may become an element of the internal layout of the train (for instance, the width of the doorway or opening from the vestibule into the saloon). Moreover, the data may not necessarily represent exactly what is happening. Although the number of one flow (e.g. alighters) may appear to reduce, simultaneous movement of another group (e.g. boarders) may make the total passenger movement rate through a wide door equally satisfactory, especially for a few seconds during the transfer of flow from alighting to boarding. However, observations show that the siting of grabpoles in the doorframes of wider doors (see Figure 9) can eliminate this potential benefit.

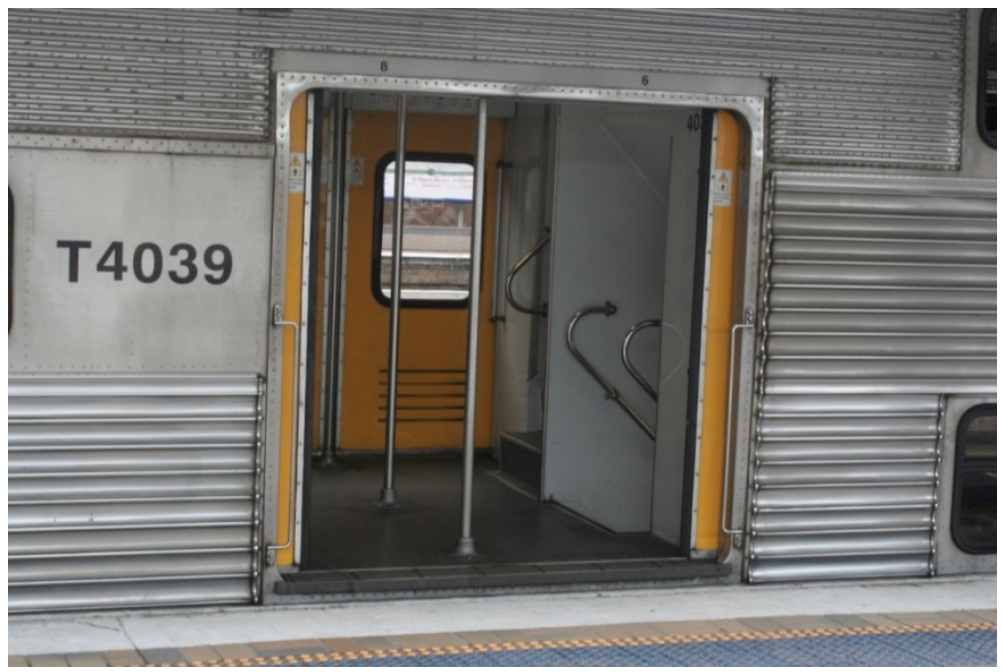

Figure 9: Grab-poles in the centre of doorframes, C stock, Sydney CityRail. 
A further complication is the presence of standbacks (space between the door hinge and the vestibule partition). These have been proven to offer substantial benefits, especially for boarders [9]. Here again, though, different uses of those standbacks make relationships potentially non-linear. Although London Underground's original work implied some discontinuity in the effect of standbacks when they were about $0.7 \mathrm{~m}$ wide, it is not necessarily the case that standbacks only provide value if wider than this. Smaller standbacks can still be useful for the storage of luggage - suitcases, for instance, are often only about $25 \mathrm{~cm}$ deep, and an ability to get this out of the way of passengers is nevertheless helpful. This is also consistent with comments made above: bigger standbacks imply larger vestibules and more space, with less impact of any interior constrictions, since these will be slightly further away, and more passengers will be able to board before such constrictions become an issue.

\section{Conclusions}

Historically, conventional wisdom has been that trains for congested urban railways (especially heavy-duty metros) should be built with wider doors to enable greater passenger flow and, indeed, the evidence shows that this is generally true. However, it is less clear that greater widths deliver the benefit expected from their greater width, as the relationships are not straightforward. Passenger flow seems to be a function of (1/width) or $\left(1 /\right.$ width $\left.^{2}\right)$, but the alighting relationship is not a smooth one. Recommendations from this research show that there are specific benefits in being $>1.25 \mathrm{~m}$ wide, and (to a lesser degree) $>1.5 \mathrm{~m}$, but very wide doors are not necessarily more efficient.

In practice, however, achieved passenger alighting rates can fall short of theoretical ones, if alighters are unable to step on to the platform if insufficient room has been left by boarders, or if boarding is restricted by constraints within the interior layout of the train. Irrespective of passenger culture, good design of trains and platforms, active platform management, and a programme of background passenger education (e.g. through announcements, platform markings and posters) are recommended to alleviate these problems.

In summary, whilst there is a demonstrably a difference in flow efficiency between single-width and double-width doors, door width per se is not the critical factor that might have been thought, because of physical constraints and typical passenger behaviours. Increased train door width does not significantly improve the rate of boarding or alighting flow, but it does have the benefit of enabling these to happen simultaneously for a few seconds.

\section{References}

[1] London Underground Ltd (1988) "Station Stop Dynamics on London Underground", Operational Research Note 88/34, June (Author: J P McKenna).

[2] Weidmann, U (1992) “Transporttechnik der Fussgänger", Schriftenreihe des IVT, Zurich, no. 90. 
[3] Weidmann, U (1994) "Der Fahrgastwechsel im öffentlichen Personenverkehr", Schriftenreihe des IVT, Zurich, no. 99.

[4] Weidmann, U (1995) “Grundlagen zur Berechnung der Fahrgastwechselzeit", Schriftenreihe des IVT, Zurich, no. 106.

[5] Heinz, W (2003) "Passenger Service Times on Trains", Licentiate Thesis, KTH, University of Stockholm.

[6] Harris, N G (2006) "Train Boarding and Alighting Rates at High Passenger Loads", Journal of Advanced Transportation 40 (3) pp. 249-263.

[7] Harris, N G \& Anderson, R J (2007) "An International Comparison of Urban Rail Boarding and Alighting Rates”, Journal of Rail \& Rapid Transit 221 F4 pp. 521-526.

[8] Harris, N G (2012) “Determinants of Profitability", 5th African Rail Safety \& Security Conference, Johannesburg, 25/07/2012.

[9] Harris, N G, Graham, D J, Anderson, R J \& Li, H (2014) "The Impact of Urban Rail Boarding and Alighting Factors", TRB, Washington DC. 